fernung (distance) anzugeben. Hier ist die Umweltpolitik unserer Auffassung nach in dringendem Zugzwang.

\section{Ökobilanzpolitik}

Nach unserer Überzeugung handelt es sich bei Aussagen, die auf der Grundlage geeigneter Ökobilanzen getroffen werden, nicht um die Diskriminierung von Produkten. Diskriminierung hieße nämlich, eine ungerechtfertigte Unterscheidung oder Ausgrenzung zu treffen. Sinn und Zweck einer seriösen Ökobilanz ist hingegen, ökologisch gerechtfertigte Differenzierungen vorzunehmen. Daher halten wir Ökobilanzen für durchaus geeignet, um Produkte zu beurteilen und gegebenenfalls auch umweltpolitische Maßnahmen einzuleiten.

Teile der Industrie scheinen hier anderer Meinung zu sein. Staatliches oder besser gesagt gesamtgesellschaftlich gewolltes Stoffstrommanagement oder eine entsprechende Produktpolitik werden abgelehnt. Aus dieser Motivation heraus werden Daten zurückgehalten, um Umweltpolitik auf der Grundlage von Ökobilanzen $\mathrm{zu}$ verhindern. Insbesondere manche im Verpackungssektor tätigen Unternehmen, vom Rohstofferzeuger über Verpackungshersteller bis zum Abfüller, sekundiert von ihren Branchenverbänden, hüten ihre Betriebsgeheimnisse gut. Ziel dieser Strategie ist die ersatzlose Demontage der Mehrwegquote in der geltenden Verpackungsverordnung. Dabei müßte allen Beteiligten klar sein, daß ,Einweg“ nicht immer schlecht, „Mehrweg“ nicht immer und für alle abzufuillenden Produkte gut ist.

Gerade eine gerechte Weichenstellung hängt von belastbaren Ökobilanzstudien ab. Wer hier Daten zurückhält, darf sich später nicht beklagen, wenn ,die Politik“ mangels Daten Rechtsnormen verabschiedet, mit denen bestimmte Verpackungssysteme diskriminiert werden.

\section{Der Autor}

Bodo M. Tegehhoff ist Diplom-Agraringenieur und seit 1990 Umweltreferent bei der Arbeitsgemeinschaft der Verbraucherverbände. Die AgV ist der Dachverband der deutschen Verbraucherorganisationen. Die Arbeitsgebiete des Autors sind Abfall, Chemikalien im Haushalt, umweltrelevante Agrarfragen, nachhaltiger Konsum.

Kontakt: AgV, Heilsbachsir. 20, 53123 Bonn, Tel. $0228 / 6489-138$, Fax 0228/644258.

Ökobilanzen und Stoffstrommanagement

\title{
Erhöhte ökologische Richtungssicherheit
}

\begin{abstract}
Die Ökobilanz wurde zu einem ausgefeilten Informationsinstrument weiterentwikkelt, doch im Hinblick auf die Eimbettung in betriebliche Entscheidungsprozesse und die Umsetzung der Ergebnisse weist sie deutliche Defizite auf. Im Umweltmanagement gewinnt wiederum die ökologische Bewertung und Optimierung von Produkten an Bedeutung, ohne groß auf Ökobilanzen zurückzugreifen. Durch eine Verbindung von Ökobilanz und Stoffstrommanagement könnten sowohl in bezug auf das Umsetzungsdefizit der Ökobilanz als auch die ökologische Richtungssicherheit von Maßnahmen des Stoffstrommanagements Verbesserungen erzielt werden.
\end{abstract}

I $n$ n den letzten Jahren wurden Ökobilanzen methodisch erheblich weiterentwickelt (1). Dabei entstand jedoch ein zunehmend schwer nachvollziehbares Expertensystem, nicht nur für Außenstehende, sondern auch für die Mehrzahl der Mitarbeiter und Mitarbeiterinnen in den beteiligten Unternehmen. Wie die komplexen und komplizierten Informationen aus Ökobilanzen innerhalb von Unternehmen in Entscheidungsprozesse der Produktentwicklung und -optimierung einfließen können, blieb bislang weitgehend unbeachtet und außerhalb der Methodenentwicklung (2). Es überrascht daher nicht, daß die Rückkopplung zum Umweltmanagement unterentwickelt ist und konkrete Verbesserungsvorschläge, sofern sie in Ökobilanzen gemacht werden, im Vergleich $\mathrm{zu}$ den vorangestellten detaillierten Berechnungen eher banal erscheinen.

Das handlungsleitende und -gestaltende Potential der Ökobilanz wird auf diese Weise nicht voll ausgeschöpft. Sinnvoll wäre die Weiterentwicklung von einem statischen zu einem prozeßbegleitenden Informationsinstrument, wobei die Umsetzung von Erkenntnissen größere Bedeutung erlangt. Ob dazu jeweils Ökobilanzen in voller Länge durchgeführt werden müssen, kann an dieser Stelle nicht näher ausgeführt werden. Diskutiert werden in diesem Zusammenhang methodische Weiterentwicklungen wie „Streamlined LCA" oder Screeningverfahren, die nach einer ersten Ökobilanz, die eine vollständige Datenbasis schafft, zum Einsatz kommen könnten (3). Durch die Arbeiten der Enquete-Kommission „Schutz des Menschen und der Umwelt" wurde das Konzept des Stoffstrommanagements ent- wickelt, das die „(...) zielorientierte, verantwortliche, ganzheitliche und effiziente Beeinflussung von Stoffströmen oder Stoffsystemen (beschreibt), wobei die Zielvorgaben aus dem ökologischen und ökonomischen Bereich kommen, unter Berücksichtigung der sozialen Aspekte. Die Ziele werden auf betrieblicher Ebene, in der Kette der an einem Stoffstrom beteiligten Akteure oder auf der staatlichen Ebene entwickelt" (4). Hier wurde also eine Methode entwickelt, die sich auf Stoff- oder Produktketten erstreckt und den Fokus auf das zwischen- und überbetriebliche Managen dieser Stoffströme legt.

Wirtschaftliche Entscheidungen werden i. d. R. unter Unsicherheit und unvollständiger Datenlage getroffen, da vollständige Informationen niemals vorliegen. Die Verfügbarkeit und Nutzung von ökologischen Informationen ist jedoch notwendig, um zielgerichtet ökologische Optimierungspotentiale identifizieren zu können und die Effektivität der getroffenen Maßnahmen im Hinblick auf die erzielten Umweltentlastungen beurteilen zu können. Die Analyse einer Vielzahl von Praxisbeispielen des Stoffstrommanagements (5) hat gezeigt, daß für den Erfolg der Stoffstrommanagementprojekte darüber hinaus die Aspekte Organisation und Motivation der beteiligten Akteure eine wesentliche Rolle spielen.

\section{Zusammenhang zwischen Ökobilanz und Stoffstrom- management}

Die These ist, daß die Effektivität von Ökobilanzen durch die systematische Einbettung in betriebliche Entscheidungsprozesse erhöht 
werden kann. Ökobilanzen beziehen den gesamten Produktlebenszyklus ein und führen damit über den Handlungsrahmen des betrieblichen Umweltmanagements hinaus zum Stoffstrommanagement, das mehrere über den Stoffstrom verbundene Akteure berücksichtigt. Der Zusammenhang zwischen Ökobilanz und Stoffstrommanagement kann vereinfacht wie folgt beschrieben werden: Auf der einen Seite gibt es ein Informationssystem, das der Einbettung in organisatorische Strukturen und Abläufe bedarf, um die erzielten Ergebnisse umsetzen zu können. Auf der anderen Seite existiert eine Managementmethode, die im Hinblick auf einen zielgerichteten und effizienten Einsatz durch Informationen über den Produktlebenszyklus verbessert werden kann.

Die idealtypische und ,genormte" Vorgehensweise bei Ökobilanzen ist, daß zunächst die Ziele und der Verwendungszweck der Studie, die Zielgruppe, der Untersuchungsrahmen und das methodische Vorgehen festgelegt werden. Darauf folgt die Datenerhebung über den gesamten Produktlebenszyklus in der Sachbilanz, dann die Zuordnung dieser Stoff- und Energieflüsse zu potentiellen Umweltwirkungen in der Wirkungsabschätzung und zuletzt eine abschließende Interpretation und Auswertung (vgl. Beitrag von Rubik, Scholl in diesem Heft). Folgt man dem Modell des Stoffstrommanagements, lassen sich folgende typische Phasen beschreiben (6):

- Initiierung

- Gestaltung

- Umsetzung/operative Phase

- Kontrolle

Ökobilanz und Stoffstrommanagement ergänzen sich nicht auf allen Stufen: Im Stoffstrommanagement sind ökologische Informationen vor allem in den Phasen Initiierung, Gestaltung und Kontrolle von Bedeutung; in der Ökobilanz fehlt insbesondere das Know-how zur Umsetzung der Ergebnisse durch die entsprechenden Akteure (vgl. Scholl, Nisius in diesem Heft).

Führt ein Unternehmen eine Ökobilanz durch oder gibt sie in Auftrag, sollte die Umsetzbarkeit

\section{Die Autorin}

Kathrin Ankele ist Diplom-Biologin und wissenschaft liche Mitarbeiterin des $10 \ddot{W}$ im Forschungsfeld ${ }_{\text {OO }}$ ko. logische Unternehmenspolitik".

Kontakt: IÖW, Giesebrechtsir. 13, 10629 Berlin, Tel. 030/884594-21, Fox 030/8825439, e-mail: mailbox@ioew.b.eunet.de der Erkenntnisse aus der Ökobilanz bereits zu Beginn stärker berücksichtigt werden. $\mathrm{Da}$ Ansatzpunkte i. d. R. nicht nur im eigenen Unternehmen identifiziert werden, ist eine aktive Einbindung von Lieferanten und Weiterverarbeitern von einem bestimmten Zeitpunkt an sinnvoll. Damit ist die Basis geschaffen, Optimierungen zu erzielen, die im gesamten Produktlebenszyklus auftreten können, also auch in deren Einflußbereich. Dazu ist es nicht zwingend erforderlich, daß alle Beteiligten dieselben Interessen verfolgen (dies wäre auch nicht realistisch). Doch ist verstärktes Nachdenken darüber geboten, wie in einem kooperativen Prozeß die ökologischen Schwachstellen eines Produktes verringert oder behoben werden können. Auf das Stoffstrommanagement bezogen, wäre dies die Phase der Initiierung.

Die Datensammlung (Sachbilanz) auf den verschiedenen Lebenszyklusstufen kann erleichtert werden, wenn Unternehmen auf den vor- und nachgelagerten Stufen nicht nur als Datenlieferanten einbezogen werden, sondern einen eigenen Nutzen aus der Ökobilanz ziehen können. Sei es, um gemeinsame Produktverbesserung zu betreiben, die Kooperation zu festigen und den Markterfolg des Produktes zu erhöhen oder eher gezwungenermaßen, um weiterhin als Zulieferer zu agieren, um nur einige mögliche Gründe zu nennen. Die Sachbilanz und die anschließende Wirkungsabschätzung dienen dazu, ökologische Schwachstellen im Produktlebenszyklus offenzulegen.

Im nächsten Schritt der Ökobilanz werden die Ergebnisse ausgewertet, interpretiert und gegebenenfalls bewertet. Nicht in jedem Fall werden Verbesserungsvorschläge sowie Ziele und Maßnahmen entwickelt, um die Schwachstellen zu beseitigen. Dies fällt ohne detaillierte Kenntnisse der organisatorischen Abläufe, der Spielräume und Restriktionen und ohne Einbeziehung der Mitarbeiter und Mitarbeiterinnen ohnehin schwer.

\section{Stärke des Stoffstrom- managements}

Hier liegt die Stärke des Stoffstrommanagements, das den Fokus auf die Gestaltung und Organisation von Prozessen und die Vereinbarung von konkreten Maßnahmen legt. Zwischen den am Verbesserungsprozeß beteiligten Unternehmen werden in intensiven Diskussionen über die Schwachstellen Lösungsmöglichkeiten entwickelt. Das Know-how der Mitarbeiter und
Mitarbeiterinnen der Unternehmen wird zur Erarbeitung von Maßnahmenvorschlägen aktiv einbezogen. In den Umweltprogrammen der Unternehmen werden diese Maßnahmen, die zuständigen Personen, der Umsetzungszeitraum sowie das zur Verfügung stehende Budget festgehalten. Ein prozeßbegleitendes Controlling unterstiitzt die Umsetzung der Maßnahmen. Diese Phase geht über den üblichen Rahmen einer Ökobilanz hinaus, die mit der Darstellung der Ergebnisse und eventuell mit der Formulierung von Vorschlägen endet. Stoffstrommanagement geht jedoch weiter, indem in der letzten Phase der Erfolg der Kooperation überprïft wird. Um den Erfolg auch in realisierter Umweltentlastung beziffern zu können, ist die ökobilanz erneut ein notwendiger Informationslieferant. Wurden die Ergebnisse nicht erreicht, müssen Korrekturmaßnahmen ergriffen werden und im Sinne eines Öko-Controlling kontinuierlich weiterverfolgt werden.

\section{Anmerkungen}

(1) DIN EN ISO 14040 (1997): Umweltmanagement. Ökobilanz, Prinzipien und allgemeine Anforderungen. Beuth.

(2) In einem Projekt des IÖW mit dem Titel „The use of LCA in business decision-making processes and its implications for environmental policy" werden erste Erfahrungen zusammengetragen, vgl. auch den Artikel von Rubik/Bültmann in diesem Heft.

(3) vgl. de Man, R.; Ankele, K.; Clous, F., Fichter; K., VölKle, E. (1997): Aufgaben des betrieblichen und betriebsübergreifenden Stoffstrommanagements. UBA-Texte

11/97, S. 67-96, Curran, M. A.; Young, S. (1996): "Report from the EPA Conference on Streamlining LCA". In: International Journal of Life Cycle Assessment, Vol. 1, Nr. 1 sowie Weitz et al. (1996): "Streamlining Life Cycle Assessment. Considerations and a Report and the State of Practice". In: International Journal of Life Cycle Assessment, Vol. 1, Nr. 2.

(4) Enquete-Kommission "Schutz des Menschen und der Umwelt" (1994): Die Industriegesellschaft gestalten - Perspektiven für einen nachhaltigen Umgang mit Stoff- und Materialströmen. Economica, S. 719 Glossar.

(5) vgl. de Man u.a. (1997) a.a.0.

6) vgl. de Man u.a. (1997) a.0.0.

Bitte beachten Sie den Wechsel unserer Anzeigenberatung. Es betreut Sie ab 1/98:

Ben Ansorge, ökom Anzeigenberatung Telefon (089) 544 184-21 Fax (089) $544184-99$ 
(c) 20I0 Authors; licensee IÖW and oekom verlag. This is an article distributed under the terms of the Creative Commons Attribution Non-Commercial No Derivates License (http://creativecommons.org/licenses/by-nc-nd/3.o/), which permits unrestricted use, distribution, and reproduction in any medium, provided the original work is properly cited. 